Pacific

Journal of

Mathematics

\title{
LOCAL ZETA FUNCTION FOR NONDEGENERATE HOMOGENEOUS MAPPINGS
}

W. A. Zuniga-GALINDO 


\title{
LOCAL ZETA FUNCTION FOR NONDEGENERATE HOMOGENEOUS MAPPINGS
}

\author{
W. A. ZunigA-GALindo
}

\begin{abstract}
We give an explicit description of the poles of the Igusa local zeta function associated to a polynomial mapping $g$, in the case in which it is a nondegenerate homogeneous mapping of degree $d$. The proof uses a generalization of the $p$-adic stationary phase formula and Néron $p$-desingularization.
\end{abstract}

\section{Introduction}

Let $K$ be nonarchimedean local field, $O_{K}$ the valuation ring of $K, P_{K}$ the maximal ideal of $O_{K}$, and $\bar{K}=O_{K} / P_{K}$ the residue field of $K$. The cardinality of the residue field of $K$ is denoted by $q$; thus $\bar{K}=\mathbb{F}_{q}$. Denote the valuation of $z \in K$ by $v(z) \in$ $\mathbb{Z} \cup\{+\infty\}$, and let $|z|_{K}=q^{-v(z)}$ and ac $z=z \pi^{-v(z)}$, where $\pi$ is a fixed uniformizing parameter for $O_{K}$. For $x=\left(x_{1}, \ldots, x_{l}\right) \in K^{l}$, set $\|x\|_{K}:=\max _{1 \leqslant i \leqslant l}\left|x_{i}\right|_{K}$.

Let $g_{i}(x) \in O_{K}\left[x_{1}, \ldots, x_{n}\right]$ be a nonconstant polynomial for $i=1, \ldots, l$, and $g(x)=\left(g_{1}(x), \ldots, g_{l}(x)\right): K^{n} \rightarrow K^{l}$ a polynomial mapping with $l \leqslant n$. Let $\chi_{i}$ be a character of $O_{K}^{\times}$, i.e., a homomorphism $\chi_{i}: O_{K}^{\times} \rightarrow \mathbb{C}^{\times}$with finite image, for $i=1, \ldots, l$. We formally put $\chi_{i}(0)=0, i=1, \ldots, l, \chi:=\left(\chi_{1}, \ldots, \chi_{l}\right)$, and define

$$
\chi(\operatorname{ac} g(x)):=\prod_{i=1}^{l} \chi_{i}\left(\operatorname{ac} g_{i}(x)\right) .
$$

The Igusa local zeta function associated to $\chi$ and $g$ is then defined as (1-1) $\quad Z(s, \chi, g):=\int_{O_{K}^{n}} \chi(\operatorname{ac} g(x))\|g(x)\|_{K}^{s}|d x|, \quad s \in \mathbb{C}$ with $\operatorname{Re} s>0$, where $|d x|$ denotes the Haar measure on $K^{n}$ normalized so $O_{K}^{n}$ has measure 1 .

Local zeta functions of type (1-1) were introduced by Weil [1965] and their basic properties for general $g$ and $l=1$ were first studied by Igusa. Using resolution of singularities Igusa proved that $Z(s, \chi, g)$ admits a meromorphic continuation to the complex plane as a rational function of $q^{-s}$ [Igusa 1978; 2000, Theorem 8.2.1]. His proof was generalized by Meuser [1981, Theorem 1] to the case $l \geqslant 1$. Using $p$-adic cell decomposition, Denef [1984] gave a completely different proof of the rationality of $Z\left(s, \chi_{\text {triv }}, g\right)$, with $l \geqslant 1$.

MSC2000: 11S40, 11D79,

Keywords: local zeta function, polynomial mappings, $p$-adic stationary formula, congruences in many variables. 
We write $Z(s, g)=Z\left(s, \chi_{\text {triv }}, g\right)$ when $\chi(z)=\chi_{\text {triv }}(z)=(1, \ldots, 1)$. Let $N_{m}(g)$ be the number of solutions of

$$
g_{i}(x) \equiv 0 \bmod P_{K}^{m}, i=1, \ldots, l, \text { in }\left(O_{K} / P_{K}^{m}\right)^{n},
$$

and set $P(t, g):=\sum_{m=0}^{\infty} N_{m}(g)\left(q^{-n} t\right)^{m}$, with $N_{0}(g)=1$. By [Meuser 1981, Theorem 2], the Poincaré series $P(t, g)$ is related to $Z(s, g)$ by the formula

$$
P(t, g)=\frac{1-t Z(s, g)}{1-t}, \quad t=q^{-s} .
$$

Thus the rationality of $Z(s, g)$ implies that of $P(t, g)$.

Igusa's local zeta functions are related to the number of solutions of congruences $\bmod \pi^{m}$ and exponential sums mod $\pi^{m}$. Indeed, in the case $l=1$, Igusa showed that the largest pole of $Z(s, g)$ controls the asymptotic behavior of number of solutions of (1-2), and the largest pole of $Z(s, \chi, g)$ controls the asymptotic behavior absolute value of $\sum_{x \bmod \pi^{m}} \Psi(\lambda g(x))$, where $\Psi$ is an standard additive character. See [Denef 1991; Igusa 1978; 1974; 1975; 2000]. Motivated by this work, Igusa asked [1978, p. 32] how one could extend his method and results to the case of polynomial mappings. The theory of local zeta functions for the case $l>1$ is just starting, and only some partial results have been obtained; see [Denef 1984; 2000; Lichtin 2000; Meuser 1981; Zuniga-Galindo 2004].

The first result of this paper, Theorem 2.3, is a generalization of the $p$-adic stationary phase formula to polynomial mappings. Igusa has used the $p$-adic stationary phase formula to compute the local zeta functions of several classes of prehomogeneous vectors spaces (see [Igusa 1994] and the references therein); the author has used this formula and Néron $p$-desingularization [Artin 1969] in the study and computation of local zeta functions associated to several classes of polynomials [Zuniga-Galindo 2001; 2003a; 2003b]. Section 2 of this paper includes applications of the generalized formula to the calculation of certain integrals are included in.

Our second main result, Theorem 3.1, provides an explicit description of the poles of $Z(s, g, \chi)$, with $1 \leqslant l \leqslant n$, in the case where $g$ is a nondegenerate homogeneous mapping of degree $d$ (Definition 3.1). The proof uses Theorem 2.3 and Néron $p$-desingularization. Still in Section 3 we prove some consequences of this description of the poles of $Z(s, g, \chi)$.

\section{The $p$-adic Stationary Phase Formula for Mappings}

In this section we give a generalization of the $p$-adic stationary phase formula (abbreviated SPF), and compute some integrals that will be used in the next section. The results about the SPF given here constitute a generalization of some results of [Zuniga-Galindo 2001, Section 2] and [Zuniga-Galindo 2003b, Section 2]. 
Some p-adic integrals. If $\xi$ is an element of $O_{K}$, we denote by $\bar{\xi}$ its image under the canonical homomorphism $O_{K} \rightarrow O_{K} / \pi P_{K}=\mathbb{F}_{q}$, i.e., the reduction of $\xi$ modulo $\pi$. If $g=\left(g_{1}, \ldots, g_{l}\right): K^{n} \rightarrow K^{l}$, where $l \leqslant n$, is a polynomial mapping with $g_{i}(x) \in O_{K}\left[x_{1}, \ldots, x_{n}\right] \backslash P_{K}\left[x_{1}, \ldots, x_{n}\right], i=1, \ldots, l$, we denote by $\bar{g}:=\left(\bar{g}_{1}, \ldots, \bar{g}_{l}\right): \mathbb{F}_{q}^{n} \rightarrow \mathbb{F}_{q}^{l}$ its reduction modulo $\pi$. We fix a lifting $R$ of $\mathbb{F}_{q}^{n}$ in $O_{K}^{n}$, and if $\xi \in R$ satisfies $\xi=\bar{\xi} \bmod \pi$, we say that $\xi$ is the lifting of $\bar{\xi}$.

Given $\xi_{0} \in O_{K}$, we define the dilatation of $g_{i}(x)$ at $\xi_{0}$ as

$$
g_{i, \xi_{0}}(x):=\pi^{-e_{i, \xi_{0}}} g_{i}\left(\xi_{0}+\pi x\right),
$$

where $e_{i, \xi_{0}}$ is the minimum order of $\pi$ in the coefficients of $g_{i}\left(\xi_{0}+\pi x\right)$.

If $y=\left(y_{1}, \ldots, y_{M}\right) \in K^{M}$ and $z=\left(z_{1}, \ldots, z_{N}\right) \in K^{N}$, we set

$$
\|(y, z)\|_{K}:=\left\|\left(y_{1}, \ldots, y_{M}, z_{1}, \ldots, z_{N}\right)\right\|_{K} .
$$

Proposition 2.1. Let $h(x)=\left(h_{1}(x), \ldots, h_{N}(x)\right)$ and $g(x)=\left(g_{1}(x), \ldots, g_{M}(x)\right)$ be polynomial mappings with $h_{j}(x), g_{i}(x) \in O_{K}\left[x_{1} \ldots, x_{n}\right]$ for $j=1, \ldots, N$, $i=1, \ldots, M$, and $N \geqslant 1, M \geqslant 1$. Let

$I\left(s, \chi, h, g, c_{0}\right):=\int_{O_{K}^{n}} \chi(\operatorname{ac} h(x))\left\|\left(c_{0}, g(x)\right)\right\|_{K}^{s}|d x| \quad$ for $s \in \mathbb{C}$ with $\operatorname{Re} s>0$, where $\chi=\left(\chi_{1}, \ldots, \chi_{N}\right)$ and $c_{0}$ a nonzero element of $O_{K}$. Then $I\left(s, \chi, h, g, c_{0}\right)$ is a polynomial in $q^{-s}$ with complex coefficients.

Proof. We work by induction on $\alpha\left(I\left(s, \chi, h, g, c_{0}\right)\right)=\alpha:=\max \left(v\left(c_{0}\right), M\right)$. The proof of the case $\alpha=1$ involves two subcases: (I) $v\left(c_{0}\right)=0, M=1$, and (II) $v\left(c_{0}\right)=1, c_{0}=\pi u, u \in O_{K}^{\times}, M=1$. The first follows immediately; the second is proved as follows:

$$
\begin{aligned}
I\left(s, \chi, h, g, c_{0}\right) & =\int_{O_{K}^{n}} \chi(\operatorname{ach}(x))\|(\pi u, g(x))\|_{K}^{s}|d x| \\
& =\sum_{\xi \in R} q^{-n} \int_{O_{K}^{n}} \chi(\operatorname{ach}(\xi+\pi x))\|(\pi u, g(\xi+\pi x))\|_{K}^{s}|d x|,
\end{aligned}
$$

with

$$
\begin{aligned}
q^{-n} \int_{O_{K}^{n}} \chi(\operatorname{ach} h(\xi+\pi x))\|(\pi u, g(\xi+\pi x))\|_{K}^{s}|d x| & \\
= & \begin{cases}q^{-n-s} \int_{O_{K}^{n}} \chi(\operatorname{ac} h(\xi+\pi x))|d x| & \text { if } \bar{g}(\bar{\xi})=0, \\
q^{-n} \int_{O_{K}^{n}} \chi(\operatorname{ac} h(\xi+\pi x))|d x| & \text { if } \bar{g}(\bar{\xi}) \neq 0 .\end{cases}
\end{aligned}
$$

Suppose that the result is valid for every integral of form $(2-1)$ with $1 \leqslant \alpha \leqslant k$, $k \in \mathbb{N}$. Let $I\left(s, \chi, h, g, c_{0}\right)$ be an integral of form (2-1), with $\alpha=k+1$. Set

$$
V:=\left\{\xi \in O_{K}^{n} \mid \bar{\xi} \in \bar{g}^{-1}(0)\right\},
$$


$I=\{1, \ldots, M\}, J \subseteq I$, and

$$
V_{J}:=\left\{\xi \in O_{K}^{n} \mid v\left(g_{i}(\xi)\right)=0 \Longleftrightarrow i \in J\right\} .
$$

Denote by $\bar{V}, \bar{V}_{J}$ the images of $V, V_{J}$ under the canonical homomorphism $O_{K}^{n} \rightarrow$ $\left(O_{K} / \pi P_{K}\right)^{n}=\mathbb{F}_{q}^{n}$. With this notation $O_{K}^{n}$ can be partitioned as

$$
O_{K}^{n}=V \bigcup \bigcup_{\substack{J \subseteq I \\ J \neq \varnothing}} V_{J},
$$

and from this it follows that

$$
\int_{O_{K}^{n}}=\int_{V}+\sum_{\substack{J \subseteq I \\ J \neq \varnothing}} \int_{V_{J}} .
$$

Therefore the proof of the case $\alpha=k+1$ is reduced to showing that

$$
\int_{V} \chi(\operatorname{ach}(x))\left\|\left(c_{0}, g(x)\right)\right\|_{K}^{s}|d x| \in \mathbb{C}\left[q^{-s}\right],
$$

and

$$
\int_{V_{J}} \chi(\operatorname{ac} h(x))\left\|\left(c_{0}, g(x)\right)\right\|_{K}^{s}|d x| \in \mathbb{C}\left[q^{-s}\right] \quad \text { for nonempty } J \subseteq I .
$$

To prove (2-2) we proceed as follows. By decomposing $V$ into equivalence classes modulo $\pi$ we obtain

$(2-4)$

$$
\begin{aligned}
& \int_{V} \chi(\operatorname{ach}(x))\left\|\left(c_{0}, g(x)\right)\right\|_{K}^{s}|d x| \\
& =\sum_{\xi \in \bar{V}} q^{-n-\gamma s} \int_{O_{K}^{n}}\left(\prod_{i=1}^{N} \chi_{i}\left(\operatorname{ach} h_{i, \xi}(x)\right)\right)\left\|\left(\pi^{-\gamma} c_{0},\left(\pi^{e_{j, \xi}-\gamma} g_{i, \xi}(x)\right)_{i \in I}\right)\right\|_{K}^{s}|d x|,
\end{aligned}
$$

where $\xi$ is the lifting of $\bar{\xi}$ and $\gamma=\min \left(e_{1, \xi}, \ldots, e_{M, \xi}, v\left(c_{0}\right)\right)$. Since $\bar{g}_{i}(\bar{\xi})=0$ for $i=1, \ldots, M$, and $v\left(c_{0}\right) \geqslant 1$, it follows that $\gamma>1$. Equation (2-2) follows from (2-4) by the induction hypothesis because each integral in the right side of (2-4) has an $\alpha \leqslant k$.

The proof of (2-3) is as follows. By decomposing $V_{J}$ into equivalence classes modulo $\pi$ we get

$$
\begin{aligned}
(2-5) \quad \int_{V_{J}} & \chi(\operatorname{ach}(x))\left\|\left(c_{0}, g(x)\right)\right\|_{K}^{s}|d x| \\
& =\sum_{\xi \in \overline{V_{J}}} q^{-n} \int_{O_{K}^{n}}\left(\prod_{i=1}^{N} \chi_{i}\left(\operatorname{ac} h_{i, \xi}(x)\right)\right)\left\|\left(c_{0},\left(\pi^{e_{j, \xi}} g_{j, \xi}(x)\right)_{j \in I \backslash J}\right)\right\|_{K}^{s}|d x| .
\end{aligned}
$$


Since $J \neq \varnothing$, it follows that the cardinality of $I \backslash J$ is less than $M$. Now (2-3) follows from (2-5) by the induction hypothesis because each integral in the righthand side of (2-5) has an $\alpha \leqslant k$.

Given $c=\left(c_{1}, \ldots, c_{l}\right)$ a nonzero element of $O_{K}^{l}$, we set

$$
I(s)=I(s, \chi, c):=\int_{O_{K}^{l}} \prod_{i=1}^{l} \chi\left(\operatorname{ac} x_{i}\right)\left\|\left(c_{1} x_{1}, \ldots, c_{l} x_{l}\right)\right\|_{K}^{s}|d x|
$$

for $s \in \mathbb{C}$ with $\operatorname{Re} s>0$.

Proposition 2.2. With the preceding notation,

$$
I(s, \chi, c)=\left\{\begin{array}{cl}
0 & \text { if } \chi \neq \chi_{\text {triv }}, \\
\frac{L\left(q^{-s}, \chi\right)}{\left(1-q^{-l-s}\right)} & \text { if } \chi=\chi_{\text {triv }}
\end{array}\right.
$$

where $L\left(q^{-s}, \chi\right)$ is a polynomial in $q^{-s}$ with complex coefficients.

Proof. If $\chi \neq \chi_{\text {triv }}$ there exists at least one index, say 1 , such that $\chi_{1} \neq \chi_{\text {triv }}$. Let $u$ be an element of $O_{K}^{\times}$such that $\chi_{1}(u) \neq 1$. Since $I(s, \chi, c)\left(1-\chi_{1}(u)\right)=0$, it follows that $I(s, \chi, c)=0$.

If $\chi=\chi_{\text {triv }}$, we set $I\left(s, \chi_{\text {triv }}, c\right)=I(s, c)$. For a nonempty subset $J \subseteq I=$ $\{1,2, \ldots, l\}$, we set

$$
W_{J}:=\left\{x \in O_{K}^{l} \mid v\left(x_{i}\right)=0 \Longleftrightarrow i \in J\right\}
$$

and

$$
I_{J}(s, c):=\int_{W_{J}}\left\|\left(c_{1} x_{1}, \ldots, c_{l} x_{l}\right)\right\|_{K}^{s}|d x| \text { for } s \in \mathbb{C} \text { with } \operatorname{Re} s>0 .
$$

On the other hand, for each $l \geqslant 1, O_{K}^{l}$ admits the partition

$$
O_{K}^{l}=\left(P_{K}\right)^{l} \bigcup \underset{\substack{J \subseteq\{1, \ldots, l\} \\ J \neq \varnothing}}{\bigcup} W_{J},
$$

where $W_{J}$ is defined in (2-6). From this partition it follows that

$$
I(s, c)=\int_{\left(P_{K}\right)^{l}}\left\|\left(c_{1} x_{1}, \ldots, c_{l} x_{l}\right)\right\|_{K}^{s}|d x|+\sum_{\substack{J \subseteq\{1, \ldots, l\} \\ J \neq \varnothing}} I_{J}(s, c) .
$$

On the other hand, a direct calculation shows that

$$
\int_{\left(P_{K}\right)^{l}}\left\|\left(c_{1} x_{1}, \ldots, c_{l} x_{l}\right)\right\|_{K}^{s}|d x|=q^{-l-s} I(s, c)
$$

thus in order to prove the result it is sufficient to show that $I_{J}(s, c)$ is a polynomial in $q^{-s}$ for every $J \neq \varnothing$. To do so we proceed as follows. By renaming the variables, we may assume that $J=\{1,2, \ldots, f\}$, with $f \leqslant l$. Since $\left\|\left(c_{1} x_{1}, \ldots, c_{l} x_{l}\right)\right\|_{K}=\left\|\left(c_{0}, c_{f+1} x_{f+1}, \ldots, c_{l} x_{l}\right)\right\|_{K} \quad$ for any $\left(x_{1}, \ldots, x_{l}\right) \in W_{J}$, 
where $c_{0}$ is an element of $O_{K}$ satisfying $\left|c_{0}\right|_{K}=\max _{1 \leqslant i \leqslant f}\left|c_{i}\right|_{K}$, we obtain

$$
I_{J}(s, c)=\left(1-q^{-1}\right)^{f} \int_{O_{K}^{l-f}}\left\|\left(c_{0}, c_{f+1} x_{f+1}, \ldots, c_{l} x_{l}\right)\right\|_{K}^{s}|d x| .
$$

Now the result follows by applying Proposition 2.1 to $I_{J}(s, c)$.

The p-adic stationary phase formula. For $A \subseteq O_{K}^{n}$, denote by $Z_{A}(s, \chi, F)$ the integral $\int_{A} \chi(\operatorname{ac} F(x))\|F(x)\|_{K}^{s}|d x|$. Let $L$ be an arbitrary field and consider a polynomial mapping $g: L^{n} \rightarrow L^{l}$, where $l \leqslant n$. Let

$$
\mathscr{g}_{g}(z):=\left(\frac{\partial g_{i}}{\partial x_{j}}\right)_{\substack{1 \leqslant i \leqslant l \\ 1 \leqslant j \leqslant n}}(z)
$$

be the Jacobian matrix of $g$ at $z \in L^{n}$, and $\mathscr{C}_{g}(L)$ the $L$-critical set of $g$ :

$$
\mathscr{C}_{g}(L)=\left\{z \in L^{n} \mid \operatorname{rank}_{L} \mathscr{F}_{g}(z) \leqslant l-1\right\} .
$$

Finally, put $\operatorname{Sing}_{g}(L):=\mathscr{C}_{g}(L) \cap g^{-1}(0)$.

The next result is a generalization of the $p$-adic stationary phase formula (see [Igusa 2000, Theorem 10.2.1], for example) to the current setting.

Theorem 2.3. Let $f_{i}(x) \in O_{K}\left[x_{1}, \ldots, x_{n}\right] \backslash P_{K}\left[x_{1}, \ldots, x_{n}\right]$ be a nonconstant polynomial for $i=1, \ldots, l$, with $l \leqslant n$, and set $F^{*}(x)=\left(f_{1}(x), \ldots, f_{l}(x)\right)$. Let $c_{i}, i=1, \ldots, l$, be nonzero elements of $O_{K}$ such that at least one $c_{i}$ belongs to $O_{K}^{\times}$, and set $F(x)=\left(c_{1} f_{1}(x), \ldots, c_{l} f_{l}(x)\right)$. Let $\bar{E}$ be a nonempty subset of $\mathbb{F}_{q}^{n}$, $\bar{S}=\operatorname{Sing}_{\bar{F}^{*}}\left(\mathbb{F}_{q}\right) \cap \bar{E}$, and $E, S$ the preimages of $\bar{E}$ and $\bar{S}$ under $O_{K}^{n} \rightarrow\left(O_{K} / P_{K}\right)^{n}$. Then

$$
Z_{E}(s, \chi, F)=L_{0}\left(q^{-s}, \chi, F\right)+\frac{L_{1}\left(q^{-s}, \chi, F\right)}{\left(1-q^{-l-s}\right)}+Z_{S}(s, \chi, F),
$$

where $L_{0}\left(q^{-s}, \chi, F\right)$ and $L_{1}\left(q^{-s}, \chi, F\right)$ are polynomials in $q^{-s}$ with complex coefficients and degrees independent of $\chi$. Moreover $L_{1}\left(q^{-s}, \chi\right)=0$ if $\chi \neq \chi$ triv.

Proof. Since $E \backslash S$ and $S$ form a partition of $E$,

$$
Z_{E}(s, \chi, F)=Z_{E \backslash S}(s, \chi, F)+Z_{S}(s, \chi, F) .
$$

Thus it is sufficient to show that

$$
Z_{E \backslash S}(s, \chi, F)=L_{0}\left(q^{-s}, \chi, F\right)+\frac{L_{1}\left(q^{-s}, \chi, F\right)}{\left(1-q^{-l-s}\right)} .
$$

By decomposing $E \backslash S$ into equivalence classes modulo $\pi$, we check that

$$
Z_{E \backslash S}(s, \chi, F)=\sum_{\bar{\xi} \in \bar{E} \backslash \bar{S}} q^{-n} \int_{O_{K}^{n}} \chi(\operatorname{ac} F(\xi+\pi x))\|F(\xi+\pi x)\|_{K}^{s}|d x| .
$$


We first consider the contribution of the points $\xi \in E \backslash S$ satisfying $\bar{F}^{*}(\bar{\xi}) \neq 0$. This condition is equivalent to the existence of a nonempty subset $T=T_{\xi}$ of $I=$ $\{1, \ldots, l\}$ such that

$$
\bar{f}_{i}(\bar{\xi}) \neq 0 \Longleftrightarrow i \in T
$$

From (2-8) it follows that

$$
\left\|\left(c_{1} f_{1}(\xi+\pi x), \ldots, c_{l} f_{l}(\xi+\pi x)\right)\right\|_{K}=\left\|\left(c_{0},\left(c_{i} f_{i}(\xi+\pi x)\right)_{i \in I \backslash T}\right)\right\|_{K}
$$

for any $x \in O_{K}^{n}$, where $c_{0}$ is an element of $O_{K}$ satisfying $\left|c_{0}\right|_{K}=\max _{i \in T}\left|c_{i}\right|_{K}$.

Thus the contribution of an $\xi \in E \backslash S$ satisfying (2-8) is

$$
q^{-n} \int_{O_{K}^{n}} \prod_{i=1}^{l} \chi_{i}\left(\operatorname{acc} c_{i} f_{i}(\xi+\pi x)\right)\left\|\left(c_{0},\left(c_{i} f_{i}(\xi+\pi x)\right)_{i \in I \backslash T}\right)\right\|_{K}^{s}|d x| .
$$

By Proposition 2.1 the preceding integral is a polynomial $L_{\bar{\xi}}\left(q^{-s}, \chi\right)$. By adding all these polynomials we obtain

$$
L_{0}\left(q^{-s}, \chi, F\right)=\sum_{\substack{\bar{\xi} \in \bar{E} \backslash \bar{S} \\ F^{*}(\bar{\xi}) \neq 0}} L_{\bar{\xi}}\left(q^{-s}, \chi, F\right) .
$$

Now we consider the contribution of points $\xi \in E \backslash S$ satisfying $\bar{F}^{*}(\bar{\xi})=0$. This condition implies that the Jacobian matrix of $\bar{F}^{*}$ at $\bar{\xi}$ has rank $l$.

Set

$$
y_{i}=\phi_{i}(x)=\left\{\begin{array}{cl}
\frac{f_{i}(\xi+\pi x)-f_{i}(\xi)}{\pi} & \text { for } i=1, \ldots, l, \\
x_{i} & \text { for } i=l+1, \ldots, n .
\end{array}\right.
$$

By the nonarchimedean implicit function theorem $y=\phi(x):=\left(\phi_{1}(x), \ldots, \phi_{n}(x)\right)$ gives a measure-preserving mapping from $O_{K}^{n}$ to $O_{K}^{n}$. By performing a change of variables from the $x_{i}$ to the $y_{i}$ one can check that

$$
\begin{aligned}
q^{-n} \int_{O_{K}^{n}} \chi(\operatorname{ac} F(\xi+\pi x)) \| F(\xi & +\pi x) \|_{K}^{s}|d x| \\
& =q^{-n-s} \int_{O_{K}^{l}} \prod_{i=1}^{l} \chi\left(\operatorname{ac} c_{i} y_{i}\right)\left\|\left(c_{i} y_{i}\right)_{1 \leqslant i \leqslant l}\right\|_{K}^{s}|d y| .
\end{aligned}
$$

By Proposition 2.2, the integral in this equation is a rational function of the form

$$
\frac{R_{\bar{\xi}}\left(q^{-s}\right)}{\left(1-q^{-l-s}\right)},
$$

and the polynomial $R_{\bar{\xi}}\left(q^{-s}\right)$ vanishes if $\chi \neq \chi_{\text {triv }}$. By adding all these polynomials we get

$$
L_{1}\left(q^{-s}, \chi\right)=\sum_{\substack{\bar{\xi} \in \bar{E} \backslash \bar{S} \\ F^{*}(\bar{\xi})=0}} R_{\bar{\xi}}\left(q^{-s}, \chi\right)
$$


Néron p-desingularization. We now use techniques of Néron $p$-desingularization (see [Artin 1969, Sect. 4], for example) together with the SPF to compute certain $p$ adic integrals. The results presented here are generalizations of results in [ZunigaGalindo 2001, Section 2] and [Zuniga-Galindo 2003b, Section 2].

Let $g_{i}(x) \in O_{K}\left[x_{1}, \ldots, x_{n}\right] \backslash P_{K}\left[x_{1}, \ldots, x_{n}\right]$ be a nonconstant polynomial for $i=1, \ldots, l$ and let $g(x)=\left(g_{1}(x), \ldots, g_{l}(x)\right): K^{n} \rightarrow K^{l}$ be a polynomial mapping with $l \leqslant n$. We denote by $S(g)$ the subset of $R$ mapped bijectively to the set $\operatorname{Sing}_{\bar{g}}\left(\mathbb{F}_{q}\right)$ by the canonical homomorphism $O_{K}^{n} \rightarrow\left(O_{K} / P_{K}\right)^{n}$. For simplicity we say that $S(g)$ is the lifting of $\operatorname{Sing}_{\bar{g}}\left(\mathbb{F}_{q}\right)$.

Given $\xi_{0} \in S(g)$, we define the dilatation of $g_{i}(x)$ at $\xi_{0}$ as

$$
g_{i, \xi_{0}}(x):=\pi^{-e_{i, \xi_{0}}} g_{i}\left(\xi_{0}+\pi x\right),
$$

where $e_{i, \xi_{0}}$ is the minimum order of $\pi$ in the coefficients of $g_{i}\left(\xi_{0}+\pi x\right)$. We say that $S\left(g_{i, \xi_{0}}\right)$, the lifting of $\operatorname{Sing}_{\bar{g}_{i, \xi_{0}}}\left(\mathbb{F}_{q}\right)$, is the first generation of descendants of $\xi_{0}$. Given a sequence of points $\left(\xi_{k}\right)_{k \in \mathbb{N}}$ in $O_{K}^{n}$ with $\xi_{0} \in S(g)$, we define inductively $e_{i, \xi_{0}, \ldots, \xi_{k}}, g_{i, \xi_{0}, \ldots, \xi_{k}}(x)$, and $S\left(g_{i, \xi_{0}, \ldots, \xi_{k}}\right)$ by setting

$$
g_{i, \xi_{0}, \ldots, \xi_{k}}(x):=\pi^{-e_{i}, \xi_{0}, \ldots, \xi_{k}} g_{i, \xi_{0}, \ldots, \xi_{k-1}}\left(\xi_{k}+\pi x\right) \text { for } k \geqslant 1,
$$

where $\xi_{k}$ lies in $S\left(g_{\left.i, \xi_{0}, \ldots, \xi_{k-1}\right)}\right)$ and $e_{i, \xi_{0}, \ldots, \xi_{k}}$ is the minimum order of $\pi$ in the coefficients of $g_{i, \xi_{0}, \ldots, \xi_{k-1}}\left(\xi_{k}+\pi x\right)$. The $(k+1)$-st generation of descendants of $\xi_{0}$ is by definition the set

$$
S\left(g_{i, \xi_{0}, \ldots, \xi_{k}}\right)=\bigcup_{\xi_{k} \in S\left(f_{\xi_{0}, \ldots, \xi_{k-1}}\right)} S\left(g_{i, \xi_{0}, \ldots, \xi_{k-1}, \xi_{k}}\right) .
$$

These definitions extend to polynomial mappings as follows. Given a sequence of points $\left(\xi_{k}\right)_{k \in \mathbb{N}}$ in $O_{K}^{n}$, with $\xi_{0} \in S(g)$, we define inductively $g_{\xi_{0}, \ldots, \xi_{k}}(x)$ and $S\left(g_{\xi_{0}, \ldots, \xi_{k}}\right)$ by setting

$$
g_{\xi_{0}, \ldots, \xi_{k}}(x):=\left(g_{1, \xi_{0}, \ldots, \xi_{k}}(x), \ldots, g_{l, \xi_{0}, \ldots, \xi_{k}}(x)\right) \text { for } k \geqslant 0,
$$

where $\xi_{k} \in S\left(g_{\xi_{0}, \ldots, \xi_{k-1}}\right)$ and $g_{i, \xi_{0}, \ldots, \xi_{k}}(x)$ is the dilatation of $g_{i, \xi_{0}, \ldots, \xi_{k-1}}(x)$ at $\xi_{k}$. The set

$$
S\left(g_{\left.i, \xi_{0}, \ldots, \xi_{k}\right):=} \bigcup_{\xi_{k} \in S\left(g_{\xi_{0}, \ldots, \xi_{k-1}}\right)} S\left(g_{\xi_{1}, \ldots, \xi_{k-1}, \xi_{k}}\right)\right.
$$

is called the $(k+1)$-th generation of descendants of $\xi_{0}$.

Remark 2.1. With the hypothesis of Theorem 2.3, we have

$$
S=\bigcup_{\bar{\xi} \in \overline{S\left(F^{*}\right)} \cap \bar{E}}\left(\xi+\pi O_{K}^{n}\right),
$$

and then

$$
Z_{S}(s, \chi, F)=\sum_{\bar{\xi} \in \overline{S\left(F^{*}\right)} \cap \bar{E}} q^{-n-\alpha_{\xi}} \int_{O_{K}^{n}} \prod_{i=1}^{l} \chi_{i}\left(\operatorname{ac} f_{i, \xi}(x)\right)\left\|\left(\pi^{e_{i, \xi}-\alpha_{\xi}} f_{i, \xi}(x)\right)_{1 \leqslant i \leqslant l}\right\|_{K}^{s}|d x|,
$$


where $\alpha_{\xi}:=\min _{i}\left\{e_{i, \xi}\right\}$. Therefore the stationary phase formula can be rewritten as

$$
\begin{aligned}
Z_{E}(s, \chi, F) & =L_{0}\left(q^{-s}, \chi, F\right)+\frac{L_{1}\left(q^{-s}, \chi, F\right)}{\left(1-q^{-l-s}\right)} \\
& +\sum_{\bar{\xi} \in \overline{S\left(F^{*}\right)} \cap \bar{E}} q^{-n-\alpha_{\xi}} \int_{O_{K}^{n}} \prod_{i=1}^{l} \chi_{i}\left(\operatorname{ac} f_{i, \xi}(x)\right)\left\|\left(\pi^{e_{i, \xi}-\alpha_{\xi}} f_{i, \xi}(x)\right)_{1 \leqslant i \leqslant l}\right\|_{K}^{s}|d x| .
\end{aligned}
$$

Let $g_{i} \in O_{K}\left[x_{1}, \ldots, x_{n}\right] \backslash P_{K}\left[x_{1}, \ldots, x_{n}\right]$, for $i=1, \ldots, l \leqslant n$, be nonconstant polynomials. Set $g(x)=\left(g_{1}(x), \ldots, g_{l}(x)\right): K^{n} \rightarrow K^{l}$, and let $\xi$ be a point of $O_{K}^{n}$ such that $\xi \notin \operatorname{Sing}_{g}(K)$. Define

$$
\mathscr{L}(g, \xi)=\min _{M}\{v(M(\xi))\},
$$

where $M$ runs over the minors of the Jacobian matrix $\mathscr{g}_{g}(\xi)$ of $g$ at $\xi$.

Proposition 2.4. With the notation just defined:

(1) If $\xi \in O_{K}^{n}$ and $\xi \in \bar{g}^{-1}(0)$, then

$$
\mathscr{L}(g, \xi)=0 \Longleftrightarrow \xi \notin \operatorname{Sing}_{\bar{g}}\left(\mathbb{F}_{q}\right) .
$$

(2) If $A \subseteq O_{K}^{n}$ is an open and compact subset such that $A \cap \operatorname{Sing}_{g}(K)=\varnothing$, there exists a constant $c(g, A) \in \mathbb{N}$ such that

$$
\sup _{\xi \in A} \mathscr{L}(g, \xi) \leqslant c(g, A) .
$$

Proof. Part (1) follows from the Jacobian criterion. Part (2) is proved by contradiction. If $\mathscr{L}(g, \xi)$ is not bounded on $A$, there exists a sequence $\left(\xi_{i}\right)_{i \in \mathbb{N}}$ of points of $A$ such that $\mathscr{L}\left(g, \xi_{i}\right) \rightarrow \infty$ when $i \rightarrow \infty$. Since $A$ is compact the sequence $\left(\xi_{i}\right)_{i \in \mathbb{N}}$ has a limit point $\xi^{*}$ in $A$. Thus, since $\operatorname{Sing}_{g}(K)$ is closed, $\xi^{*}$ lies in $A \cap C_{g}(K)$; that is, $A \cap \operatorname{Sing}_{g}(K) \neq \varnothing$, contradicting $A \cap \operatorname{Sing}_{g}(K)=\varnothing$.

The index $\mathscr{L}(g, \xi)$ appears naturally associated to SPF, as already noted in [Zuniga-Galindo 2001]. It plays a central role in the construction of the Néron $p$-desingularization of the special fiber of smooth schemes over $\operatorname{Spec}\left(O_{K}\right)$; see, for example, [Artin 1969, Section 4].

Lemma 2.5. Let $g_{i} \in O_{K}\left[x_{1}, \ldots, x_{n}\right] \backslash P_{K}\left[x_{1}, \ldots, x_{n}\right]$, for $i=1, \ldots, l \leqslant n$, be nonconstant polynomials. Set $g(x)=\left(g_{1}(x), \ldots, g_{l}(x)\right): K^{n} \rightarrow K^{l}$. Let $A \subseteq O_{K}^{n}$ be an open and compact subset such that $A \cap \operatorname{Sing}_{g}(K)=\varnothing$. Take $\xi_{0} \in A$.

(1) Suppose that $\xi_{k}$, for some $k \geqslant 0$, satisfies

$$
\xi_{k} \in \begin{cases}S(g) & \text { if } k=0, \\ S\left(g_{\xi_{0}, \ldots, \xi_{k-1}}\right) & \text { if } k \geqslant 1,\end{cases}
$$

where $\xi_{1}, \ldots, \xi_{k-1}$ are descendants of $\xi_{0}$, and that $\xi_{k}$ has at least one descendant in $S\left(g_{\xi_{0}, \ldots, \xi_{k}}\right)$. Then

$$
\mathscr{L}\left(g_{\xi_{0}, \ldots, \xi_{k}}, 0\right) \leqslant \mathscr{L}\left(g, \xi_{0}+\xi_{1} \pi+\cdots+\xi_{k} \pi^{k}\right)-(k+1) .
$$


(2) For any $\xi_{0} \in A \cap S(g)$, if $k \geqslant c(g, A)+1$, then $S\left(g_{i, \xi_{0}, \ldots, \xi_{k}}\right)=\varnothing$.

Proof. (1) For each $j=0,1, \ldots, k$, we take $\xi_{j+1} \in S\left(g_{i, \xi_{0}, \ldots, \xi_{j}}\right), e_{i, \xi_{0}, \ldots, \xi_{j}}$ as in (2-9), (2-10), and define

$$
E_{i, k}:=\sum_{j=0}^{k} e_{i, \xi_{0}, \ldots, \xi_{j}}
$$

Thus $E_{i, k} \geqslant k+1$ for every $i$. With the above notation one checks that

$$
g_{i}\left(\xi_{0}+\xi_{1} \pi+\cdots+\xi_{k} \pi^{k}+\pi^{k+1} x\right)=\pi^{E_{i, k}} g_{i, \xi_{1}, \ldots, \xi_{k-1}, \xi_{k}}(x) .
$$

It follows that

$$
\mathscr{L}\left(g, \xi_{0}+\xi_{1} \pi+\cdots+\xi_{k} \pi^{k}\right) \geqslant \sum_{i=1}^{l}\left(E_{i, k}-k-1\right)+\mathscr{L}\left(g_{\xi_{0}, \ldots, \xi_{k-1}, \xi_{k}}, 0\right) .
$$

Since $\left(E_{i, k}-k-1\right) \geqslant 0$ for every $i$, this inequality implies

$$
\mathscr{L}\left(g, \xi_{0}+\xi_{1} \pi+\cdots+\xi_{k} \pi^{k}\right) \geqslant E_{1, k}-k-1+\mathscr{L}\left(g_{\xi_{0}, \ldots, \xi_{k-1}, \xi_{k}}, 0\right) .
$$

The result follows from this inequality by showing that $E_{1, k} \geqslant 2(k+1)$. This last assertion follows from

$$
e_{1, \xi_{0}, \ldots, \xi_{j}} \geqslant 2 \text { for } j=0,1, \ldots, k,
$$

which we prove as follows. Let $\xi_{j} \in O_{K}^{n}$, for $j=0,1, \ldots, k$, represent a point satisfying the conditions of part (1) of the lemma. We write

$$
\begin{aligned}
g_{i, \xi_{1}, \ldots, \xi_{j-1}}\left(\xi_{j}+\pi x\right)=g_{i, \xi_{1}, \ldots, \xi_{j-1}}\left(\xi_{j}\right)+\pi \sum_{j=1}^{n} \frac{\partial g_{i, \xi_{1}, \ldots, \xi_{j-1}}}{\partial x_{j}}\left(\xi_{j}\right) x_{j} \\
\quad+\pi^{2}(\text { terms of degree } \geqslant 2)
\end{aligned}
$$

for $i=1, \ldots, l$. From $(2-12)$ it follows that

$$
v\left(g_{i, \xi_{1}, \ldots, \xi_{j-1}}\left(\xi_{j}\right)\right) \geqslant 1,
$$

and there follows also the existence of a minor $M\left(\xi_{j}\right)$ of the Jacobian matrix of $g_{i, \xi_{1}, \ldots, \xi_{j-1}}$ at $\xi_{j}$ such that

$$
\mathscr{L}\left(g_{\xi_{0}, \ldots, \xi_{j-1}}, \xi_{j}\right)=v\left(M\left(\xi_{j}\right)\right) \geqslant 1 .
$$

Without loss of generality we may suppose that

$$
M\left(\xi_{j}\right)=\operatorname{det}\left(\frac{\partial g_{i}}{\partial x_{j}}\right)_{\substack{1 \leqslant i \leqslant l \\ 1 \leqslant j \leqslant l}}\left(\xi_{j}\right) .
$$

Thus the rank of $\bar{M}\left(\bar{\xi}_{j}\right)$ over $\mathbb{F}_{q}$ is less than $l$. By taking an invertible linear transformation $\Omega: O_{K}^{n} \rightarrow O_{K}^{n}$, with entries in $O_{K}$, we may assume that, say,

$$
\frac{\partial g_{1, \xi_{1}, \ldots, \xi_{j-1}}}{\partial x_{j}}\left(\xi_{j}\right)=\pi a_{1, j} \quad \text { for all } j \text {. }
$$


Therefore

(2-13) $g_{1, \xi_{1}, \ldots, \xi_{j-1}}\left(\xi_{j}+\pi x\right)=\pi a_{1,0}+\pi^{2} \sum_{j} a_{1, j} x_{j}+\pi^{2}$ (terms of degree $\geqslant 2$ ).

At the same time, the lemma's condition that $\xi_{k}$ has at least one descendant in $S\left(g_{\xi_{0}, \ldots, \xi_{k}}\right)$ implies that the congruence

$$
g_{1, \xi_{1}, \ldots, \xi_{j-1}}\left(\xi_{j}+\pi x\right) \equiv 0 \bmod \pi^{2}
$$

has a solution in $R$. This fact and (2-13) imply that $a_{1,0} \equiv 0 \bmod \pi$; therefore

$$
g_{1, \xi_{1}, \ldots, \xi_{j-1}}\left(\xi_{j}+\pi x\right)=\pi^{e_{1, \xi_{0}, \ldots, \xi_{j}}} g_{1, \xi_{1}, \ldots, \xi_{j}}(x),
$$

with $e_{1, \xi_{0}, \ldots, \xi_{j}} \geqslant 2$ for $j=0,1, \ldots, k$.

Part (2) of the lemma follows immediately from Part (1).

Lemma 2.6. Let $E \subseteq O_{K}^{n}$ be the preimage under the canonical homomorphism $O_{K}^{n} \longrightarrow\left(O_{K} / \pi O_{K}\right)^{n}$ of a subset $\bar{E} \subseteq \mathbb{F}_{q}^{n}$. Let $g(x)=\left(g_{1}(x), \ldots, g_{l}(x)\right): K^{n} \rightarrow K^{l}$ be a polynomial mapping such that $g_{i}(x) \in O_{K}\left[x_{1}, \ldots, x_{n}\right] \backslash P_{K}\left[x_{1}, \ldots, x_{n}\right]$ for $i=1, \ldots, l, l \leqslant n$, and suppose $\operatorname{Sing}_{g}(K) \cap E=\varnothing$. Then

$$
Z_{E}(s, \chi, g)=\frac{L\left(q^{-s}, \chi, g\right)}{1-q^{-l-s}}
$$

where $L\left(q^{-s}, \chi, g\right)$ is a polynomial in $q^{-s}$ with complex coefficients.

Proof. We define inductively $I_{k}$ as follows:

$$
\begin{aligned}
& I_{1}=S(g) \cap E, \\
& I_{k}=\left\{\left(\xi_{1}, \ldots, \xi_{k}\right) \mid\left(\xi_{1}, \ldots, \xi_{k-1}\right) \in I_{k-1} \text { and } \xi_{k} \in S\left(f_{\xi_{1}, \ldots, \xi_{k-1}}\right)\right\} \quad \text { for } k \geqslant 2 .
\end{aligned}
$$

We set $E\left(\xi_{1}, \ldots, \xi_{k}\right):=\alpha_{\xi_{1}}+\alpha_{\xi_{1}, \xi_{2}}+\cdots+\alpha_{\xi_{1}, \xi_{2}, \ldots, \xi_{k}}$. If $m=c(g, E)+1$, then $I_{m+1}=\varnothing$, because part (2) of Lemma 2.5 implies that $S\left(f_{\xi_{1}, \xi_{2}, \ldots, \xi_{m}}\right)=\varnothing$ for every $\left(\xi_{1}, \xi_{2}, \ldots, \xi_{m}\right) \in I_{m}$. The result follows by applying the stationary phase formula $m+1$-times:

$$
\begin{aligned}
Z_{E}(s, \chi, g)= & L_{0}(E, \bar{g}, \chi)+\frac{L_{1}(E, \bar{g}, \chi)}{\left(1-q^{-l-s}\right)} \\
& +\sum_{k=1}^{m} q^{-k n}\left(\sum_{\left(\xi_{1}, \ldots, \xi_{k}\right) \in I_{k}} L_{0}\left(\bar{g}_{\xi_{1}, \ldots, \xi_{k}}, O_{K}^{n}, \chi\right) q^{-E\left(\xi_{1}, \ldots, \xi_{k}\right) s}\right) \\
& +\frac{1}{\left(1-q^{-l-s}\right)} \sum_{k=1}^{m} q^{-k n}\left(\sum_{\left(\xi_{1}, \ldots, \xi_{k}\right) \in I_{k}} L_{1}\left(\bar{g}_{\xi_{1}, \ldots, \xi_{k}}, O_{K}^{n}, \chi\right) q^{-E\left(\xi_{1}, \ldots, \xi_{k}\right) s}\right) .
\end{aligned}
$$




\section{Local zeta functions of homogeneous nondegenerate mappings}

In this section we state and prove the second main result of this paper (Theorem 3.1) and some of its consequences.

Definition 3.1. A polynomial mapping $g(x)=\left(g_{1}(x), \ldots, g_{l}(x)\right): K^{n} \rightarrow K^{l}$ is called a nondegenerate homogeneous mapping of degree $d$ if:

(1) $g_{i}(x) \in O_{K}\left[x_{1}, \ldots, x_{n}\right]$ is a nonconstant homogeneous polynomial of degree $d$, for $i=1, \ldots, l$;

(2) $l \leqslant n$;

(3) $\operatorname{Sing}_{g}(K) \bigcap\left(K^{\times}\right)^{n}=\varnothing$.

Theorem 3.1. Let $g(x)=\left(g_{1}(x), \ldots, g_{l}(x)\right): K^{n} \rightarrow K^{l}$ be a nondegenerate homogeneous mapping of degree $d$. Then

$$
Z(s, \chi, g)=\frac{L\left(q^{-s}, \chi, g\right)}{\left(1-q^{-n-d s}\right)\left(1-q^{-l-s}\right)},
$$

where $L\left(q^{-s}, \chi, g\right)$ is a polynomial in $q^{-s}$ with complex coefficients.

Proof. By partitioning $O_{K}^{n}$ as $\left(P_{K}\right)^{n} \cup W$, with

$$
W:=\left\{\xi \in O_{K}^{n} \mid v\left(\xi_{i}\right)=0 \text {, for some } i\right\},
$$

we check that

$$
\begin{aligned}
Z(s, \chi, g) & =Z_{\left(P_{K}\right)^{n}}(s, \chi, g)+Z_{W}(s, \chi, g) \\
& =q^{-n-d s} Z(s, \chi, g)+Z_{W}(s, \chi, g) .
\end{aligned}
$$

The result follows by applying Lemma 2.6 to $Z_{W}(s, \chi, g)$.

Corollary 3.2. For $i=1, \ldots$, , let $g_{i}(x) \in O_{K}\left[x_{1}, \ldots, x_{n}\right]$ be a nonconstant homogeneous polynomial of degree $d$, and assume $g(x)=\left(g_{1}(x), \ldots, g_{l}(x)\right)$ : $K^{n} \rightarrow K^{l}$ is a nondegenerate homogeneous mapping of degree $d$. If

$$
N_{m}(g)=\operatorname{Card}\left(\left\{\xi \in\left(O_{K} / P_{K}^{m}\right)^{n} \mid g_{i}(\xi) \equiv 0 \bmod \pi^{m}, i=1, \ldots, l\right\}\right),
$$

then

$$
\lim \sup _{m \rightarrow \infty}\left(N_{m}\left(g_{1}, \ldots, g_{l}\right)\right)^{1 / m} \leqslant q^{n-\min (l, n / d)} .
$$

In particular $N_{m}\left(g_{1}, \ldots, g_{l}\right) \leqslant q^{m(n-\min (l, n / d))}$ for $m$ big enough.

Proof. The result follows by estimating the radius of convergence

$$
r=\frac{1}{\limsup _{m \rightarrow \infty}\left(N_{m}(g) q^{-n}\right)^{1 / m}}
$$

of the Poincaré series $P(t, g)=\sum_{m=0}^{\infty} N_{m}(g)\left(q^{-n} t\right)$. Since

$$
P(t, g)=\frac{1-t Z(s, g)}{1-t},
$$


with $t=q^{-s}$ (see [Meuser 1981, Theorem 2]), Theorem 3.1 implies that

$$
r \geqslant q^{(n-\min (l, n / d))} .
$$

The statement in the corollary is a simple reformulation of this equation.

\section{References}

[Artin 1969] M. Artin, "Algebraic approximation of structures over complete local rings", Inst. Hautes Études Sci. Publ. Math. 36 (1969), 23-58. MR 42 \#3087 Zbl 0181.48802

[Denef 1984] J. Denef, "The rationality of the Poincaré series associated to the $p$-adic points on a variety”, Invent. Math. 77:1 (1984), 1-23. MR 86c:11043 Zbl 0537.12011

[Denef 1991] J. Denef, "Report on Igusa's local zeta function”, pp. 359-386 (Exposé 741) in Séminaire Bourbaki (1990/91), Astérisque 201-203, 1991, Available at http://www.wis.kuleuven.ac.be/ algebra/denef.html. MR 93g:11119 Zbl 0749.11054

[Denef 2000] J. Denef, "Arithmetic and geometric applications of quantifier elimination for valued fields", pp. 173-198 in Model theory, algebra, and geometry, edited by D. Haskell et al., Math. Sci. Res. Inst. Publ. 39, Cambridge Univ. Press, Cambridge, 2000. MR 2001e:03063 Zbl 0981.03041

[Igusa 1974] J.-i. Igusa, "Complex powers and asymptotic expansions, I: Functions of certain types", J. Reine Angew. Math. 268/269 (1974), 110-130. MR 50 \#254 Zbl 0287.43007

[Igusa 1975] J.-i. Igusa, "Complex powers and asymptotic expansions, II: Asymptotic expansions", J. Reine Angew. Math. 278/279 (1975), 307-321. MR 53 \#8018 Zbl 0315.41029

[Igusa 1978] J.-i. Igusa, Forms of higher degree, Tata Lectures on Mathematics and Physics 59, Tata Institute of Fundamental Research, Bombay, 1978. MR 80m:10020 Zbl 0417.10015

[Igusa 1994] J.-i. Igusa, "A stationary phase formula for $p$-adic integrals and its applications”, pp. 175-194 in Algebraic geometry and its applications (West Lafayette, IN, 1990), edited by C. L. Bajaj, Springer, New York, 1994. MR 95a:11104 Zbl 0904.11035

[Igusa 2000] J.-i. Igusa, An introduction to the theory of local zeta functions, AMS/IP Studies in Advanced Mathematics 14, American Mathematical Society, Providence, RI, 2000. MR 2001j:11112 Zbl 0959.11047

[Lichtin 2000] B. Lichtin, "On a question of Igusa: towards a theory of several variable asymptotic expansions, I”, Compositio Math. 120:1 (2000), 25-82. MR 2001c:11086 Zbl 0969.11015

[Meuser 1981] D. Meuser, "On the rationality of certain generating functions", Math. Ann. 256:3 (1981), 303-310. MR 83g:12015 Zbl 0471.12014

[Weil 1965] A. Weil, "Sur la formule de Siegel dans la théorie des groupes classiques", Acta Math. 113 (1965), 1-87. MR 36 \#6421 Zbl 0161.02304

[Zuniga-Galindo 2001] W. A. Zúñiga Galindo, "Igusa's local zeta functions of semiquasihomogeneous polynomials", Transactions Amer. Math. Soc. 353:8 (2001), 3193-3207. MR 2001j:11116 Zbl 1007.11072

[Zuniga-Galindo 2003a] W. A. Zuniga-Galindo, "Computing Igusa's local zeta functions of univariate polynomials, and linear feedback shift registers", J. Integer Seq. 6:3 (2003), article 03.3.6, 18 pp. MR 2046406

[Zuniga-Galindo 2003b] W. A. Zuniga-Galindo, "Local zeta functions and Newton polyhedra", Nagoya Math. J. 172 (2003), 31-58. MR 2004h:11098

[Zuniga-Galindo 2004] W. A. Zuniga-Galindo, "On the poles of Igusa's local zeta function for algebraic sets”, Bull. London Math. Soc. 36:3 (2004), 310-320. MR 2038719 
Received December 27, 2002.

W. A. ZUNIGA-GALINDO

BARRY UNIVERSITY

DEPARTMENT OF MATHEMATICS ANd COMPUTER SCIENCE

11300 NE SECOND AVEnUE

MiAMI SHORES FL 33161

wzuniga@mail.barry.edu

http://Euclid.Barry.edu/ zuniga 